05

\title{
О постановке экспериментов по определению пробивной способности хвостовых участков кумулятивных струй
}

\author{
() С.В. Федоров \\ Московский государственный технический университет им. Н.Э. Баумана, \\ 105005 Москва, Россия \\ e-mail: sergfed-64@mail.ru
}

Поступило в Редакцию 2 декабря 2020 г.

В окончательной редакции 14 декабря 2020 г.

Принято к публикации 14 декабря 2020 г.

По данным расчетов пробивное действие кумулятивных зарядов может быть существенно повышено (на 40-50\% в случае преграды из высокопрочной стали), если за счет повышения точности их изготовления уменьшить нижний порог скорости, при котором происходит прекращение проникания хвостовых участков кумулятивной струи в преграду. Для экспериментального подтверждения этих данных предложено подробно исследовать пробивную способность хвостовых участков кумулятивных струй с использованием стержней-отсекателей из материала с высокой плотностью, располагаемых на небольшом расстоянии от кумулятивного заряда (менее его диаметра) и предназначенных для устранения более скоростной части струи. На основании численного моделирования в рамках двумерной осесимметричной задачи механики сплошных сред спрогнозированы возможные параметры стержней-отсекателей для получения уединенных „хвостов“ кумулятивных струй с различными скоростями лидирующего элемента.

Ключевые слова: взрыв, кумулятивный заряд, кумулятивная струя, пробивное действие, критическая скорость, эффективная скорость, стержень-отсекатель.

DOI: $10.21883 /$ JTF.2021.05.50691.331-20

\section{Введение}

Современные кумулятивные заряды (КЗ) с медной конической облицовкой кумулятивной выемки при прецизионной технологии их изготовления способны пробивать высокопрочные стальные преграды с толщиной на уровне $10 d_{0}\left(d_{0}-\right.$ диаметр заряда) [1-3]. Преграда пробивается высокоскоростной кумулятивной струей (КС), формирующейся при схлопывании облицовки под действием продуктов детонации заряда взрывчатого вещества [4,5]. Сформировавшаяся КС при движении в свободном полете вследствие существующего в ней градиента осевой скорости сначала растягивается с сохранением сплошности, а затем по причине развития пластической неустойчивости распадается на множество отдельных уже недеформирующихся (безградиентных) элементов [6-8]. Распределение скорости по длине КС обычно близко к линейному при скорости „головы“ струи на уровне $10 \mathrm{~km} / \mathrm{s}$. К числу основных факторов, влияющих на пробивное действие КС при условии ее идеальной прямолинейности, относятся плотности материалов струи и преграды, прочностные свойства преграды и длина струи $[2,3]$.

Технологические погрешности изготовления КЗ, такие, как, например, разнотолщинность облицовки в окружном направлении, некруглость поперечных сечений облицовки, разноплотность заряда взрывчатого вещества, смещение инициирующего узла от оси заряда и т. п., приводят к тому, что участки КС в момент фор- мирования при схлопывании облицовки приобретают импульс в боковом (от оси заряда) направлении $[2,9,10]$. Следствием этого является снижение пробивной способности КС, так как ее участки из-за бокового дрейфа отклоняются от строго соосного следования друг за другом (струя искривляется). В результате участки, находящиеся ближе к „хвосту“, могут столкнуться со стенками отверстия в преграде, пробитого предшествующей частью струи, и поэтому не достичь его дна и не проучаствовать в дополнительном углублении пробоины [11-14].

Для обеспечения высокого пробивного действия КЗ необходимо повышать уровень их прецизионности, что приводит к существенному удорожанию технологического процесса их изготовления. В то же время ясно, что существует предельно возможная глубина пробития преграды, которая уже не может быть превышена путем дальнейшего повышения точности изготовления КЗ. Предельно возможное пробитие КЗ обеспечивается в том случае, когда созданы условия для участия в углублении пробоины всеми способными к этому участками КС. Однако потенциальная пробивная способность различных участков КС неодинакова и для хвостовых участков со скоростями ниже $3-4 \mathrm{~km} / \mathrm{s}$ существенно зависит от их скорости, снижаясь с уменьшением последней [2]. Поэтому для рационального выбора точности изготовления КЗ необходима информация о вкладе в общую глубину пробития преграды участков КС с различными скоростями. 


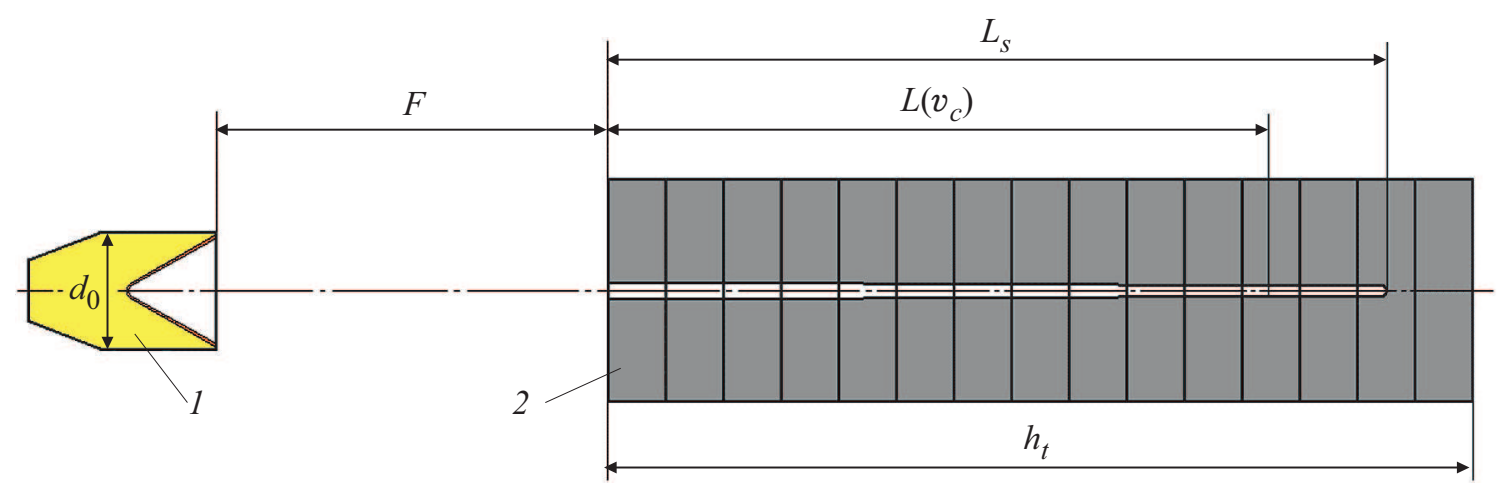

Рис. 1. Схема экспериментов по определению пробивного действия КЗ: 1 - К3; 2 - преграда из пластин с размещенными между ними электроконтактными датчиками.

\section{1. Эффективная и критическая скорости кумулятивной струи}

При экспериментальных исследованиях пробивного действия различных участков КС преграда, располагаемая на определенном удалении $F$ от КЗ (рис. 1), составляется из отдельных пластин относительно небольшой толщины, между которыми располагаются электроконтактные датчики. С помощью этих датчиков определяется время пробития каждой пластины и соответственно скорость проникания КС при пробитии пластин, находящихся на различном удалении от лицевой поверхности преграды. Скорость струи восстанавливается затем по скорости проникания ее участков обычно на основании модифицированного уравнения Бернулли [2] в пренебрежении прочностными свойствами материала КС (прочность материала струи обычно существенно меньше прочности преграды), приводящего к следующей взаимосвязи между указанными скоростями

$$
v=u+\sqrt{u^{2} / \gamma+v_{c r}^{2}},
$$

где $v$ - скорость участка КС, $u$ - скорость проникания этого участка, параметр $\gamma=\rho_{j} / \rho_{t}$ характеризует соотношение плотностей материалов струи $\rho_{j}$ и преграды $\rho_{t}$, $v_{c r}$ - критическая скорость струи, определяемая с использованием динамической твердости преграды $H_{d}$, как

$$
v_{c r}=\sqrt{2 H_{d} / \rho_{j}}
$$

и соответствующая скорости, при которой проникание КС прекращается вследствие прочностного сопротивления преграды.

По результатам проведения экспериментов в указанной постановке может быть определена зависимость $L(v)$, где $L-$ глубина пробития преграды частью КС со скоростями выше значения $v$, а также установлен нижний предел скорости для участков струи, принявших участие в пробитии, называемый эффективной скоростью КС $v_{\text {eff }}[15-17]$. Значение $v_{\text {eff }}$ всегда выше значения $v_{c r}$, так как в реальной ситуации прекращение проникания хвостовых участков КС происходит не вследствие прочностного сопротивления преграды, а изза того, что они просто не долетают до дна пробоины, „намазываясь“ на ее стенки по причине бокового дрейфа. С повышением уровня прецизионности КЗ значение $v_{\text {eff }}$ уменьшается.

Эффективная скорость КС возрастает с увеличением удаления КЗ от преграды $F$ (рис. 1) вследствие увеличения времени движения участков струи до момента начала проникания и возможности в связи с этим их большего бокового смещения от оси КЗ. В [18] проведен анализ экспериментальных зависимостей $v_{\text {eff }}(F)$, полученных разными авторами $[10,15,16]$ для прецизионных КЗ с медными коническими облицовками при их действии по высокопрочным стальным преградам и при изменении $F$ в диапазоне $2 d_{0} \leq F \leq 25 d_{0}$. Этот анализ показывает, что экстраполяция всех зависимостей $v_{\mathrm{eff}}(F)$ на $F=0$ дает для $v_{\text {eff }}$ значения на уровне $2-2.5 \mathrm{~km} / \mathrm{s}$. Закономерен вопрос, могут ли эти значения быть ассоциированными с критической скоростью КС $v_{c r}$ и если нет, то насколько они ее еще превышают. Дело в том, что расчетное определение $v_{c r}$, согласно (2), заключает в себе значительный элемент неопределенности из-за отсутствия достоверной информации по динамической твердости преград из различных материалов в условиях проникания КС.

По данным [19] для высокопрочных стальных преград твердость, определяемая в статических условиях, составляет 3.6-4.15 GPa. Для перехода от статического к динамическому значению $H_{d}$ первое обычно домножается на коэффициент динамичности, величина которого, как правило, выбирается из диапазона 1.1-1.5. При этом считается, что с повышением статических прочностных свойств стали коэффициент динамичности для нее должен снижаться [20,21]. Даже если для высокопрочной стальной преграды с твердостью в статических условиях $4.15 \mathrm{GPa}$ принять этот коэффициент равным 1.5, то для критической скорости медной КС (2) получается значение около $1.2 \mathrm{~km} / \mathrm{s}$. Оно заметно ниже результата, который можно было бы принять за $v_{c r}$ на основе анализа экспериментальных зависимостей $v_{\text {eff }}(F)$, обзор которых приведен в [18]. Из этого следует, что хвосто- 


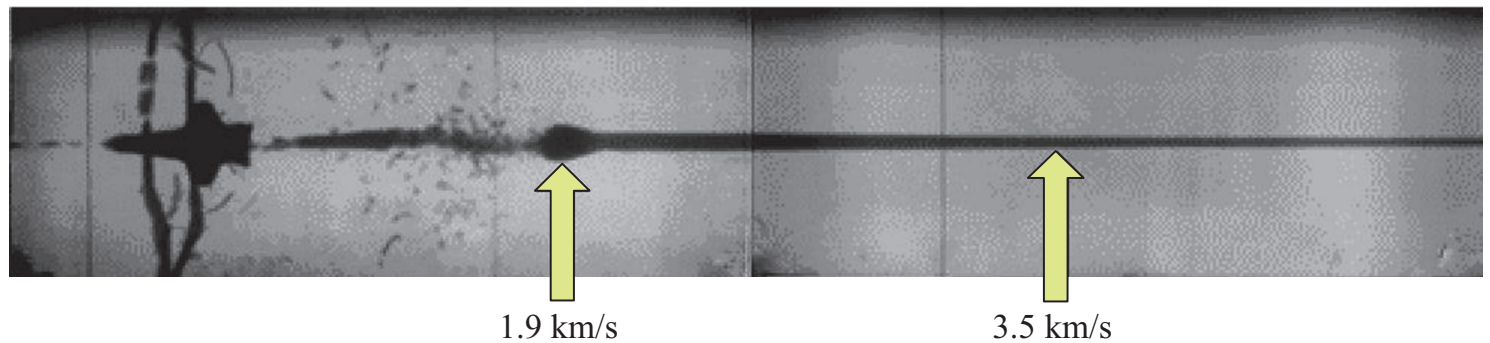

Рис. 2. Рентгенограмма хвостового участка КС.

вые участки КС со скоростью ниже $2 \mathrm{~km} / \mathrm{s}$ в случае их наличия в струе еще должны обладать пробивной способностью, для реализации которой КЗ должен иметь соответствующий уровень прецизионности. Сделанный вывод о возможных значениях критической скорости КС, очевидно, нуждается в отдельной экспериментальной проверке, так как принимавшийся при ее оценке коэффициент динамичности весьма условен в плане его соответствия условиям деформирования преграды при проникании КС.

Современные КЗ в прецизионном исполнении с медной конической облицовкой обеспечивают максимум глубины пробития преграды из высокопрочной стали на удалениях $F$ от последней $(5-7) d_{0}$. По данным $[10,15,16,18]$, при таких расстояниях К3 от преграды значения $v_{\text {eff }}$ для КС составляют не менее $3.5 \mathrm{~km} / \mathrm{s}$, и здесь уже с достоверностью можно утверждать, что они выше критической скорости и, следовательно, за счет повышения точностных характеристик КЗ можно получить дополнительный прирост его пробивного действия. Ключевое значение при этом имеет величина достигаемого прироста.

На рентгенограмме (рис. 2), любезно предоставленной О.В. Свирским, хвостовой участок КС находится еще на начальной стадии своего растяжения в отсутствие на нем каких-либо видимых признаков развития пластической неустойчивости. Длина участка струи между отмеченными сечениями со скоростями 1.9 и $3.5 \mathrm{~km} / \mathrm{s}$ составляет примерно два диаметра КЗ. К моменту распада этого участка на отдельные элементы она должна возрасти еще в несколько раз. Отсюда следует, что по критерию своей длины рассматриваемый участок КС, если исключить „помехи“ для его проникания, обусловленные боковым дрейфом, может обеспечивать весьма существенный вклад в глубину пробития КЗ (на уровне нескольких диаметров заряда).

\section{2. Потенциальная пробивная способность хвостовых участков кумулятивных струй}

Для оценки вклада в общую глубину пробития преграды участков КС с различными скоростями модифици-

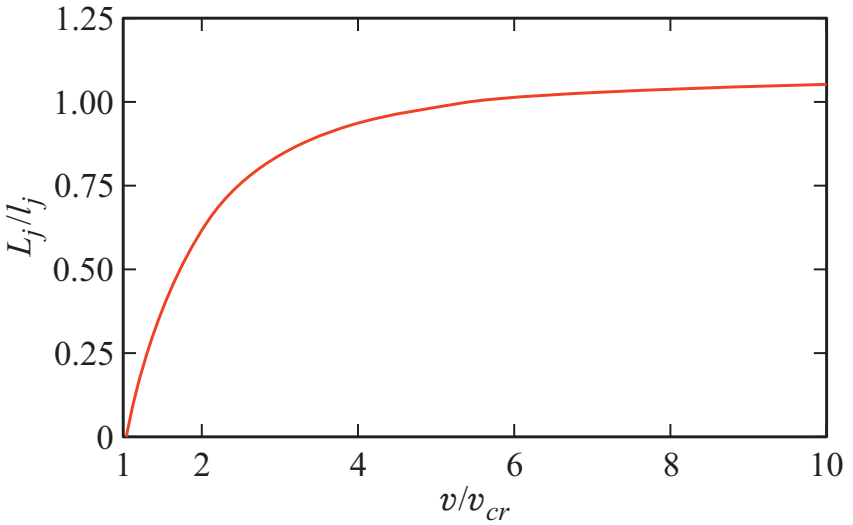

Рис. 3. Зависимость удельной пробивной способности медной КС от скорости ее участков при проникании в стальную преграду.

рованное уравнение Бернулли [2] дает соотношение

$$
\frac{L_{j}}{l_{j}}=\frac{\gamma v-\sqrt{\gamma} \sqrt{v^{2}+(\gamma-1) v_{c r}^{2}}}{\sqrt{\gamma} \sqrt{v^{2}+(\gamma-1) v_{c r}^{2}}-v},
$$

где $L_{j}$ - глубина пробития преграды участком КС длиной $l_{j}$. Как следует из (3), отношение $L_{j} / l_{j}$, характеризующее удельную пробивную способность различных участков КС, возрастает от нулевого значения при $v=v_{c r}$, стремясь при неограниченном увеличении скорости струи к постоянному значению $\sqrt{\gamma}$. На рис. 3 приводится зависимость $L_{j} / l_{j}$ от скорости участков КС при значении $\gamma$, соответствующем медной струе и стальной преграде. В области скоростей струи, близких к значению $v_{c r}$, происходит резкий рост $L_{j} / l_{j}$ с увеличением скорости. Поэтому для оценки потенциального вклада в пробитие КС ее хвостовых участков необходима возможно более достоверная информация о критической скорости струи. Например, для участка КС со скоростью $v=2.5 \mathrm{~km} / \mathrm{s}$ отношение $L_{j} / l_{j}$ составляет 0.22 при $v_{c r}=2 \mathrm{~km} / \mathrm{s}$ и возрастает почти втрое (до 0.65) при $v_{c r}=1.2 \mathrm{~km} / \mathrm{s}$.

При оценке потенциальной пробивной способности хвостовых участков КС необходимо ориентироваться не только на величину $L_{j} / l_{j}$ для них, но и на их длину в момент проникания, а она должна быть больше, чем у более скоростных участков. Во-первых, головные участ- 
ки КС могут не успеть растянуться до своей предельной длины (при которой происходит разрыв участка на отдельные безградиентные элементы) к моменту начала их проникания, „хвост“ же КС в реальной ситуации всегда проникает в разорванном состоянии, полностью исчерпав резервы дальнейшего увеличения своей эффективной длины. Во-вторых, хвостовые участки КС имеют высокие коэффициенты предельного удлинения (характеризующие степень удлинения участка на момент его разрыва) в связи с их значительным поперечным размером $[22,23]$.

Правдоподобную информацию о том, какой добавок к пробивному действию КЗ можно получить, если включить „в работу“ хвостовые участки КС, дают данные расчетов [18], проведенных на основании методики [24], учитывающей влияние на пробивное действие КЗ точности его изготовления. В указанных расчетах задавалась постоянная по длине КС скорость бокового дрейфа ее участков. Изменением величины этой скорости имитировались КЗ с различным уровнем прецизионности. Расчеты были проведены для КЗ диаметром $d_{0}=100 \mathrm{~mm}$ c медной конической облицовкой при его действии по высокопрочной стальной преграде. При скорости дрейфа участков КС $16 \mathrm{~m} / \mathrm{s}$ максимальное пробитие К3 (на оптимальном удалении от преграды) составило около $10 d_{0}$, что соответствует пробитию КЗ с обеспечиваемой в настоящее время точностью изготовления. При снижении скорости дрейфа до $8 \mathrm{~m} / \mathrm{s}$ максимальное пробитие КЗ возросло до $12 d_{0}$, а в случае „идеального“ заряда (в отсутствие дрейфа КС) оно составило $14.4 d_{0}$ (причем для „идеального“ КЗ во внимание принималось пробивное действие только участков КС со скоростью выше $2 \mathrm{~km} / \mathrm{s}$, в то время как при данной скорости, если ориентироваться на значение $v_{c r}=1.2 \mathrm{~km} / \mathrm{s}$, удельная пробивная способность струи $L_{j} / l_{j}$ еще достаточно велика и составляет 0.48). Приведенные расчетные данные свидетельствуют о том, что резервы увеличения пробивного действия КЗ за счет повышения точности их изготовления еще далеко не исчерпаны. Однако прежде, чем развертывать достаточно дорогостоящие работы по дальнейшему совершенствованию технологии изготовления К3, не излишним является экспериментальное подтверждение сделанного вывода. Детальное экспериментальное определение пробивной способности „хвостов“ КС может позволить также выбирать параметры КЗ (в первую очередь, геометрию облицовки) таким образом, чтобы происходило формирование КС с нижним пределом скорости в ее „хвосте“, равным скорости прекращения проникания (т.е., так, чтобы в струе не было „неработающих“ хвостовых участков).

\section{3. Предложения по экспериментальной проверке}

Помимо экспериментального определения критической скорости КС $v_{c r}(2)$, для рационального выбора уровня, до которого имеет смысл повышать точностные характеристики К3, интерес представляет также экспериментальное исследование пробивного действия хвостовых участков струи со скоростями, близкими к значению $v_{c r}$. В стандартных условиях постановки экспериментов (рис. 1) проникающую способность хвостовых участков КС трудно определять на фоне гораздо большего общего пробития предшествующих участков струи. Кроме того, в стандартных условиях „хвост“ КС проникает, пройдя предварительно по длинному и узкому каналу, образованному более скоростной частью струи, что вследствие бокового смещения его элементов отрицательным образом сказывается на его пробивном действии. Необходимой же является информация о глубине проникания хвостовых участков КС в отсутствие влияния этого снижающего ее фактора.

Еще одной интересной задачей для экспериментального исследования, имеющей помимо прикладного и фундаментальное значение, является сравнительный анализ пробивного действия одних и тех же хвостовых участков КС в неразорванном и разорванном состояниях. Как уже отмечалось, в реальной ситуации „хвост“ КС всегда проникает уже после его распада на отдельные элементы. Есть основания предполагать, что при проникании в разорванном состоянии участков КС со скоростями, близкими к $v_{c r}$, возникают предпосылки (являющиеся предметом отдельного обсуждения) к „затруднению“ проникания даже при строго соосном движении друг за другом безградиентных элементов, на которые разделился участок. Это может привести к прекращению проникания таких участков при скоростях, несколько превышающих скорость прекращения их проникания в неразорванном состоянии (принимаемой за $v_{c r}$ ). Фактически это означает, что причиной превышения эффективной скорости КС $v_{\text {eff }}$ над критической $v_{c r}$ может быть не только боковой дрейф участков струи, но и их проникание в разорванном состоянии. Для экспериментальной проверки данного предположения интересно было бы сравнить глубину проникания „хвоста“ КС в состоянии непосредственно перед его разделением на безградиентные элементы и в состояниях, соответствующих различным моментам после разделения, отличающихся величиной удаления безградиентных элементов друг от друга.

Главная идея постановки экспериментов по выяснению сформулированных вопросов проста и очевидна для скрупулезного исследования пробивного действия „Хвостов“ КС необходимо устранить пробитие преграды предшествующей частью струи, т. е. каким-либо образом „отсечь“ (ликвидировать) эту часть. Основное требование при этом - с хвостовой частью КС никаких изменений произойти не должно и ее геометрические и кинематические параметры должны остаться такими же, как в отсутствие указанной „отсечки“.

В [2] для получения с использованием КЗ с конической облицовкой высокоскоростного компактного элемента из „головы“ КС предлагаются различные методы „отсечки“ последующей менее скоростной части 
$a$

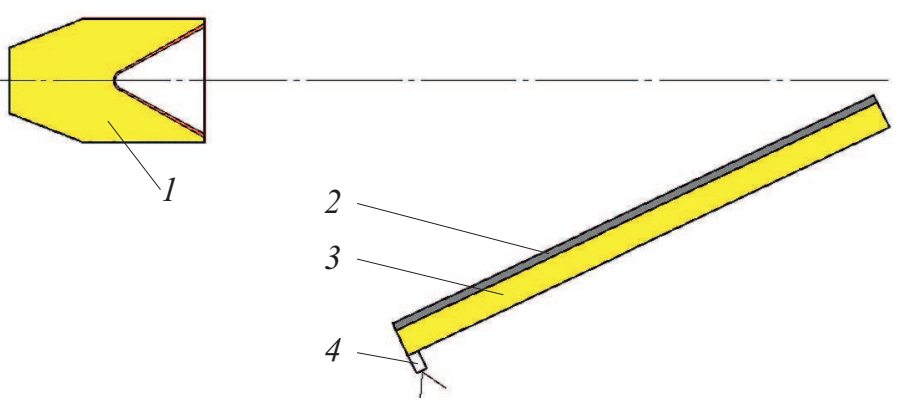

$b$

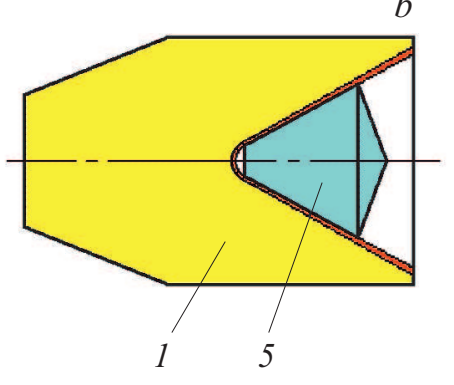

Рис. 4. Варианты „отсечки“ части КС, предшествующей хвостовому участку, с использованием метаемой взрывом металлической пластины $(a)$ и размещаемого в кумулятивной облицовке вкладыша $(b): 1-$ КЗ; $2-$ пластина; $3-$ слой взрывчатого вещества; 4 - электродетонатор; 5 - вкладыш.

струи. Два из них в принципе могут быть применены для решения рассматриваемой родственной задачи „отсечки“ головной более скоростной части КС при сохранении ее „хвоста“. В одном из методов устранение интересующей части КС осуществляется с помощью металлической пластины, метаемой слоем взрывчатого вещества сбоку на сформировавшуюся струю в свободном полете (рис. 4, $a$ ). В другом методе используется вкладыш в облицовку, препятствующий схлопыванию некоторой ее части и соответственно формированию из этой части КС (рис. $4, b)$. Однако, как представляется, при практической реализации обоих указанных методов могут возникнуть серьезные проблемы.

В методе с метанием на КС металлической пластины (рис. $4, a$ ) весьма непросто подобрать размеры пластины и синхронизировать времена инициирования КЗ и метающего пластину слоя взрывчатого вещества так, чтобы от струи оставалась только хвостовая часть с заданной скоростью ее „головы“. Ситуация дополнительно усугубляется тем, что в экспериментах необходимо будет получать „хвосты“ КС с различной скоростью лидирующего элемента, а это означает, что для каждой такой скорости нужно будет отдельно подбирать параметры метания пластины. Просматриваются еще две проблемы, которые могут возникнуть при реализации данного метода. Первая из них связана с состоянием сохраняемого хвостового участка КС после бокового удара пластины по предшествующей части струи. Велика вероятность того, что „Голова“ хвостового участка в результате такой боковой ударной „отсечки“ получит повреждения (например, искривление), которые исказят ее реальное пробивное действие. Вторая проблема заключается в том, что некоторая доля фрагментов разрушенной ударом пластины более скоростной части КС может продолжить свое движение вдоль оси КЗ к преграде. Во всяком случае, о возможности этого свидетельствуют рентгенограммы КС после их взаимодействия с метаемыми взрывом металлическими пластинами [19]. Конечно, пробивное действие этих фрагментов будет крайне слабым, но тем не менее в случае наложения оставляемых ими на преграде кратеров на пробоину от „хвоста“ КС могут быть получены искаженные результаты. Особенно недопустимым это является при экспериментальном определении критической скорости КС $v_{c r}$, так как воздействие на преграду „хвоста“ КС со скоростью лидирующего элемента, близкой к $v_{c r}$, тоже будет слабым. Следует отметить, что указанная проблема дополнительного воздействия на преграду „отсекаемой“ части КС усугубляется при инициировании детонации в метающем пластину слое взрывчатого вещества непосредственно самой КС (при размещении края пластины со слоем взрывчатого вещества на пути движения струи). В этом случае неразрушенным остается головной участок КС, успевающий „проскочить“ сквозь пробитое в пластине отверстие, пока она еще не успела набрать скорость. Пробивное действие этого участка может быть сопоставимым с пробивным действием „хвоста“ струи.

Использование вкладыша в облицовку для выделения из КС нужной ее части (рис. $4, b)$ с точки зрения практической реализации, безусловно, существенно проще метода „отсечки“, основанного на метании пластины. Однако в этом случае возникают вопросы относительно соответствия геометрических и кинематических параметров участка КС, формирующегося из остающейся „свободной“ за пределами вкладыша части облицовки у ее основания, параметрам хвостового участка КС, сформировавшегося в отсутствие вкладыша. Во-первых, условия схлопывания сечения облицовки, граничащего с вкладышем, отличны от условий схлопывания того же сечения в естественных условиях. Между основанием вкладыша и граничным сечением облицовки в процессе схлопывания может возникнуть зазор или, напротив, схлопывающаяся облицовка может начать натекать на вкладыш. В любом случае следствием этого будет искажение скоростей и углов схлопывания по сравнению с естественными условиями. Во-вторых, дополнительное изменение условий струеобразования при наличии вкладыша в облицовке сопряжено с тем, что в его отсутствие формирование хвостового участка КС происходит в уже установившемся режиме с примыканием к области струеобразования материала песта, движущегося с определенной скоростью. В присутствие же вкладыша 
струеобразование при схлопывании „свободной“ части облицовки начинается в еще неустановившемся режиме, когда позади области струеобразования либо ничего нет, либо находится неподвижный вкладыш. Ни то, ни другое не соответствует условиям струеобразования хвостового участка КС в естественных условиях. Отмеченные обстоятельства делают сомнительной возможность получения с использованием вкладыша в облицовку участка КС, полностью идентичного по всем параметрам хвостовому участку струи, формирующейся из той же облицовки в отсутствие вкладыша. Следствием же этого могут быть различия и в пробивном действии сопоставляемых участков, в том числе и за счет изменения характера их разделения на отдельные элементы. Таким образом, несмотря на привлекательность, обусловленную простотой, метод выделения из КС хвостовой части для отдельного экспериментального исследования ее пробивного действия с использованием вкладыша в облицовку (рис. 4,b) не годится для того, чтобы на основании этого исследования можно было сделать обоснованные выводы по поводу возможного прироста пробивной способности КЗ за счет включения в пробитие дополнительных участков „хвоста“ струи. Вкладыш хорошо „работает“, когда из КС нужно просто получить высокоскоростной элемент и не особенно важно, сохранятся ли у него в точности те же параметры, что у соответствующего участка КС при „свободном“ ее формировании, или нет.

С целью устранения части КС, предшествующей хвостовой, была рассмотрена возможность использования того же способа, что реализуется в стандартных условиях проведения экспериментов (рис. 1) и хорошо отработан, т. е. способа, основанного на радиальном растекании ее материала при проникании в преграду. Для решения сформулированных задач по экспериментальному исследованию пробивной способности „хвостов“ КС в этом способе требуется исключить фактор их движения по узкой пробоине в преграде и максимально возможно уменьшить толщину преграды, необходимую для ликвидации прониканием более скоростной части струи. Как оказалось, этого можно достичь, если отсекающую преграду взять в виде стержня длиной $l_{c}$ с относительно небольшим диаметром $d_{c}$ (в сравнении с диаметром К3 $d_{0}$ ) из материала с высокой плотностью $\rho_{c}$, и расположить этот стержень-отсекатель на оси КЗ на минимально допустимом расстоянии $h_{c}$ от его основания с кумулятивной выемкой (чтобы участки КС к моменту проникания в стержень успели как можно меньше удлиниться) (рис. 5). Диаметр стержня $d_{c}$ должен выбираться из условия, чтобы при пробитии КС он полностью разрушался с радиальным разлетом его материала и не мог оказать влияния на движение хвостовых участков КС даже при наличии у них скорости бокового дрейфа. Выбираемое расстояния $h_{c}$ от стержня до основания КЗ должно исключать возможность соударения фрагментов разрушающегося стержня с еще не схлопнувшейся частью кумулятивной облицовки. За счет выбора длины стержня-отсекателя $l_{c}$ можно легко управлять длиной сохраняющейся хвостовой части КС и соответственно скоростью ее лидирующего элемента $v_{c}$. За стержнемотсекателем на расстоянии $F_{c}$ от основания КЗ располагается основная преграда, в которой фиксируется пробивное действие „хвоста“ КС.

Метод „отсечки“ части КС, предшествующей ее „хвосту“, с использованием стержня-отсекателя (рис. 5) по простоте реализации не уступает методу с использованием вкладыша в облицовку (рис. 4,b), но при этом лишен основного недостатка последнего. Он „не вторгается“ в процесс формирования КС при схлопывании облицовки и гарантирует сохранение всех параметров хвостовой части струи такими же, как в экспериментах в стандартной постановке, что является необходимым условием для корректного сравнения пробивного действия „хвостов“ КС в стандартных условиях (рис. 1) и в условиях их „изоляции“ от остальной части струи.

На основании экспериментов со стержнем-отсекателем (рис. 5) видится проведение следующих конкретных исследований:

1. Определение влияния на глубину проникания хвостовых частей КС скорости их лидирующего элемента $v_{c}$ при различных удалениях КЗ от основной преграды $F_{c}$ (при проникании „хвоста“ струи в неразорванном и в разорванном состояниях). Так как глубина проникания в этих экспериментах $L_{c}$ будет гораздо меньше, чем в стандартных условиях $L_{s}$ (рис. 1 ), то и потребная толщина преграды $h_{t}$ существенно уменьшится, что, помимо облегчения проведения опытов, позволит набрать преграду из тонких пластин с более частым размещением электроконтактных датчиков для детального определения скорости проникания. На основании данных экспериментов можно будет получить адекватную информацию о критической скорости КС $v_{c r}$ и о динамической твердости материала преграды $H_{d}$ в условиях проникания КС. Получение такой информации важно не только в рамках решения рассматриваемых в настоящей работе задач. На ней в настоящее время фактически полностью базируется расчетное прогнозирование пробивного действия КС. В частности, она используется при обработке результатов стандартных экспериментов (рис. 1) на основании соотношения (1).

2. Определение, насколько сильно влияет на проникающую способность хвостовой части КС фактор ее движения в пробоине от предшествующей части струи. Для этого необходимо из экспериментов в стандартной постановке (рис. 1) иметь информацию о зависимости $L(v)$. Тогда в экспериментах с тем же КЗ со стержнем-отсекателем (рис. 5), сохраняющим хвостовую часть КС со скоростью лидирующего элемента $v_{c}$, необходимо будет расположить основную преграду на расстоянии $F_{c}=F+L\left(v_{c}\right)$ от КЗ. Сравнение полученной в этих опытах глубины проникания $L_{c}$ с длиной участка пробоины $L_{s}-L\left(v_{c}\right)$ по результатам экспериментов в стандартной постановке даст информацию о снижении пробивного действия КС вследствие бокового дрейфа ее элементов. 


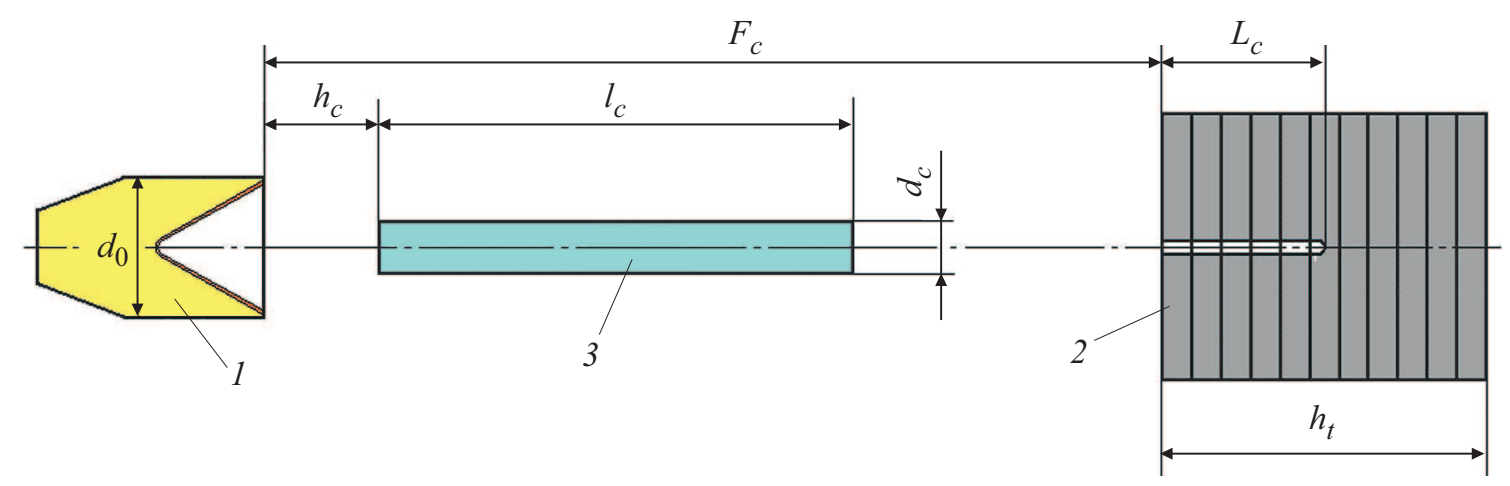

Рис. 5. Схема экспериментов по определению пробивного действия хвостовой части КС с использованием стержня-отсекателя: 1 - КЗ; 2 - преграда из пластин с размещеннными между ними электроконтактными датчиками; 3 - стержень-отсекатель.

3. Определение, как влияет на состояние хвостовой части КС фактор ее движения в пробоине от предшествующей части струи. Для этого в экспериментах в стандартной постановке (рис. 1) необходимо взять преграду толщиной $h_{t}=L\left(v_{c}\right)$ и провести рентгенографирование вышедшей из нее части струи. Полученный результат можно будет сравнить с состоянием того же хвостового участка КС со скоростью головного элемента $v_{c}$ и на том же его удалении $F+L\left(v_{c}\right)$ от КЗ при рентгенографировании этого участка в процессе его свободного движения в опыте со стержнем-отсекателем (рис. 5) в отсутствие основной преграды.

По результатам указанных исследований можно будет сделать обоснованные выводы относительно того, до каких значений имеет смысл снижать эффективную скорость КС $v_{\text {eff }}$ за счет повышения точности изготовления КЗ для увеличения его пробивного действия. Они могут быть использованы также для рационального выбора геометрических параметров кумулятивной облицовки. Рациональность состоит в том, чтобы либо исключить присутствие в формируемой КС хвостовых участков со скоростями ниже уровня, обеспечивающего их способность к прониканию, либо, напротив, добавить к „хвосту“ дополнительные участки с меньшими скоростями, обладающие пробивной способностью в случае, если скорость „хвоста“ заметно превышает этот уровень [25].

\section{4. Результаты численного моделирования}

Очевидно, ключевое значение для постановки описанных выше экспериментов имеет выбор параметров стержня-отсекателя. Для получения информации по этому вопросу было проведено численное моделирование пробития КС стержней из разных материалов в рамках двумерной осесимметричной задачи механики сплошных сред. Для проведения расчетов использовалась компьютерная программа моделирования взрывных и ударных процессов ЭРУДИТ [26-29], разработанная в МГТУ им. Н.Э. Баумана и реализующая вычислительный алгоритм свободных лагранжевых точек. Поведение мате- риалов кумулятивной облицовки и стержня-отсекателя описывалось на основании модели сжимаемой упругопластической среды с условием пластичности Мизеса при постоянном значении динамического предела текучести [30].

В расчетах был рассмотрен тот же КЗ диаметром $d_{0}=100 \mathrm{~mm}$ с медной конической облицовкой с углом раствора $60^{\circ}$, что выбирался для анализа пробивного действия в работах $[18,31]$. Стержень-отсекатель полагался цилиндрическим с диаметром $d_{c}=0.3 d_{0}$ и варьировавшейся длиной $l_{c}$. Его обращенный к КЗ торец предполагался удаленным от заряда на расстояние $h_{c}=0.75 d_{0}$. В качестве материала стержня были рассмотрены сталь (с плотностью $\rho_{c}=7850 \mathrm{~kg} / \mathrm{m}^{3}$ ), свинец $\left(\rho_{c}=11340 \mathrm{~kg} / \mathrm{m}^{3}\right)$ и тяжелый сплав на основе вольфрама ВНЖ-90 $\left(\rho_{c}=17170 \mathrm{~kg} / \mathrm{m}^{3}\right)$ [32], содержащий в своем составе вольфрам, никель и железо в массовых долях соответственно 90, 7 и $3 \%$.

Рассматриваемый КЗ формирует КС со скоростью „головы“ около $10 \mathrm{~km} / \mathrm{s}$. На рис. 6 приведен вид хвостовых участков КС на момент полного пробития стержнейотсекателей длиной $l_{c}=2.5 d_{0}$ из разных материалов $\left(v_{z}\right.$ - осевая скорость материала на оси симметрии; координата $z$ вдоль оси заряда отсчитывается от его основания с кумулятивной выемкой). Видно, что стальной стержень „убирает“ головную часть КС только со скоростями выше $v_{c} \approx 4.3 \mathrm{~km} / \mathrm{s}$, свинцовый позволяет снизить $v_{c}$ примерно до $3.3 \mathrm{~km} / \mathrm{s}$, а стержень из сплава ВНЖ-90 - до $2.6 \mathrm{~km} / \mathrm{s}$. Видно также, что все стержни „хорошо“ разлетаются в радиальном направлении, не создавая каких-либо опасностей для беспрепятственного движения сохраняемой части КС даже при наличии у нее скорости бокового дрейфа. Более подробно динамика радиального разлета материала стержня-отсекателя длиной $2.5 d_{0}$ в процессе его пробития КС проиллюстрирована на примере свинцового стержня на рис. 7.

Наиболее важную в практическом отношении информацию, касающуюся выбора длины стержня $l_{c}$, дают представленные на рис. 8 зависимости текущей скорости лидирующего элемента $v_{c}$ сохраняющейся части КС от дистанции $l_{c}^{*}$, пройденной струей в стержне (расстояния от лицевого торца стержня до текущего поло- 

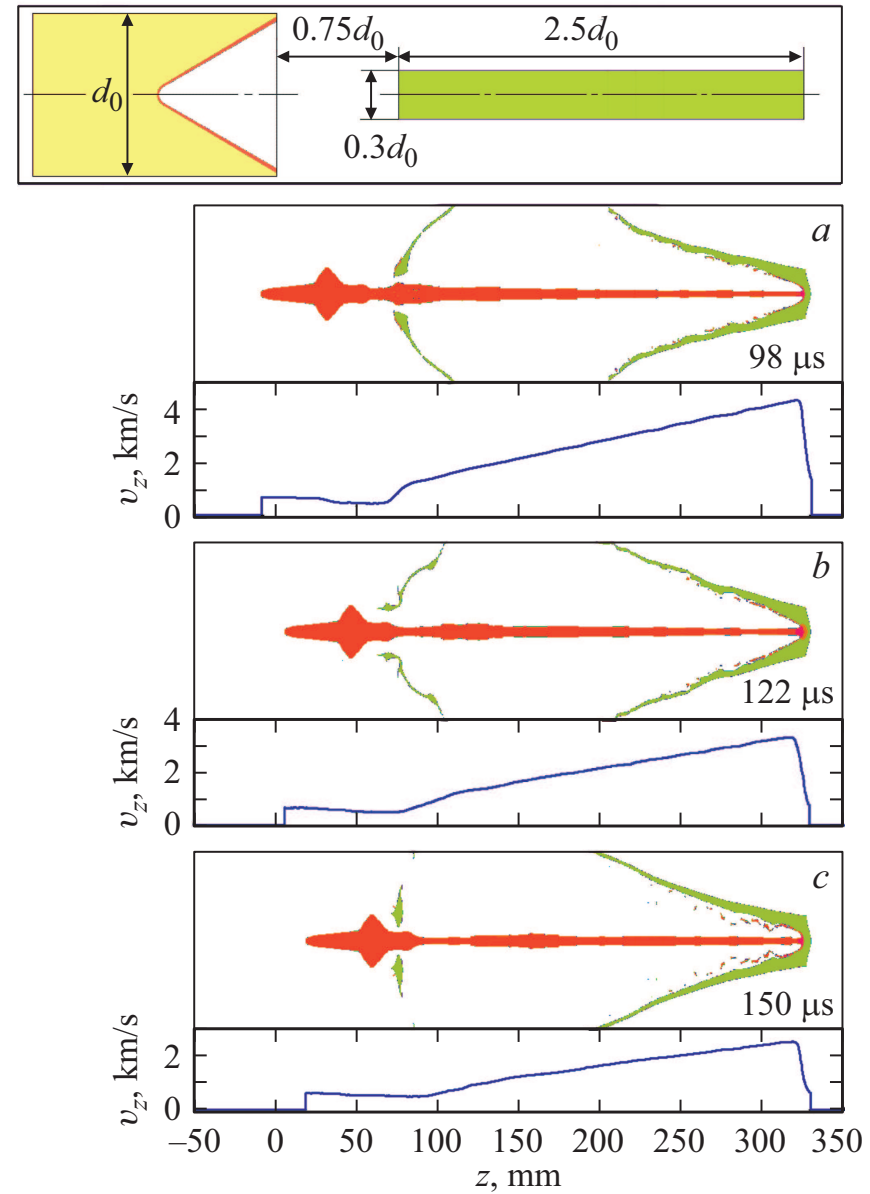

Рис. 6. Хвостовые части КС, сохраняющиеся на момент полного пробития стержней-отсекателей длиной $2.5 d_{0}$ из стали $(a)$, свинца $(b)$ и тяжелого сплава ВНЖ-90 (c) (указанные времена полного пробития отсчитываются от момента инициирования КЗ).

жения контактной границы между струей и стержнем на его оси), полученные по результатам расчетов со стержнями длиной $l_{c}=4.5 d_{0}$. Эти зависимости позволяют спрогнозировать длину стержня-отсекателя, необходимую для получения хвостовой части КС с заданным значением скорости $v_{c}$ в „голове“ этой части. Потребная длина стержня $l_{c}$ должна выбираться примерно на $(0.2-0.25) d_{0}$ большей значения $l_{c}^{*}$, определяемого по кривым на рис. 8 при заданной скорости $v_{c}$. Эта добавка учитывает тыльные эффекты, облегчающие проникание КС в стержень и соответственно замедляющие темп ее расходования в процессе проникания, когда она приближается к его дальнему от КЗ торцу. Величиной $l_{c}^{*}$ влияние тыльных эффектов игнорируется в силу специфики ее определения, как текущей глубины внедрения КС в достаточно длинный стержень. Указанная выше величина добавки была оценена на основании сопоставления значений $l_{c}^{*}$, соответствующих на рис. 8 скоростям $v_{c}$ для стержня длиной $l_{c}=2.5 d_{0}$ (рис. 6), с самим этим значением $2.5 d_{0}$. Очевидно, что если потребная скорость в „хвосте“ КС $v_{c}$ такова, что определяемое для нее на основании кривых на рис. 8 значение $l_{c}^{*}$ получается меньшим $4.5 d_{0}$ (т. е. длины стержня, задававшейся в расчетах по определению $\left.l_{c}^{*}\right)$ не более, чем на $(0.2-0.25) d_{0}$, то корректировать $l_{c}^{*}$ добавкой, учитывающей проявление тыльных эффектов, уже не следует, полагая в этом случае $l_{c}=l_{c}^{*}$.

Согласно гидродинамической теории $[1,2]$, глубина проникания КС в преграду определяется соотношением плотностей материалов струи и преграды. Поэтому можно было бы ожидать, что уменьшение потребной длины стержня-отсекателя с увеличением плотности его материала (при обеспечении одной и той же скорости „головы“ отсекаемой хвостовой части КС) будет согласованным с возрастанием соотношения плотностей материалов стержней. Однако по данным проведенных расчетов обнаруживаются некоторые отклонения в выполнении этого предположения. Соотношение плотностей составляет 1.44 в случае свинцового и стального стержней и 1.51 в случае стержней из сплава ВНЖ-90 и свинца (т. е. в первом случае оно несколько ниже, чем во втором). При этом из рис. 8 видно, что при одних и тех же обеспечиваемых значениях $v_{c}$ переход от стального стержня к свинцовому приводит, напротив, к заметно большему уменьшению потребной длины стержня, чем последующий переход от свинцового стержня к стержню из сплава ВНЖ-90. Как представляется, отмеченный эффект аномально высокой „отсекающей“ способности свинцового стержня, не коррелирующей с „правилом“ плотностей, связан с заметно большей, чем у стали и сплава ВНЖ-90, сжимаемостью свинца (низкой скоростью звука в свинце), что создает дополнительное сопротивление прониканию КС [33,34].

Проведенный сравнительный анализ „отсекающей“ способности стержней из разных материалов свидетельствует о целесообразности использования в экспериментах по исследованию пробивного действия „хвостов“ КС стержней-отсекателей из свинца или сплава ВНЖ-90. Из данных на рис. 8 с учетом сделанных замечаний относительно определения $l_{c}$ по $l_{c}^{*}$ следует, что для выделения хвостового участка КС со значением $v_{c}=2 \mathrm{~km} / \mathrm{s}$, при котором по данным $[10,15,16,18]$ проникания медной струи в высокопрочную стальную преграду в стандартных условиях (рис. 1) заведомо не происходит, потребная длина составляет $l_{c} \approx 3.0 d_{0}$ для стержня из сплава ВНЖ-90, $l_{c} \approx 4.0 d_{0}$ для свинцового стержня и $l_{c}>4.5 d_{0}$ - для стального. Это означает, что при использовании стержней из свинца или сплава ВНЖ-90 уже на расстоянии не более $(4.0-5.0) d_{0}$ от КЗ можно получить „изолированную“ хвостовую часть КС со скоростью лидирующего элемента $2 \mathrm{~km} / \mathrm{s}$ и с достоверностью установить, обладает она пробивной способностью или нет. Если ориентироваться на скорость в „голове“ сохраняемой хвостовой части КС $v_{c}=3 \mathrm{~km} / \mathrm{s}$, то возможные дистанции ее „отсечки“ (отсчитываемые от основания КЗ) стержнями из свинца или сплава ВНЖ-90 сокращаются до (3.0-3.5) $d_{0}$. По данным разных авторов [6-8,22,23,35] расстояние от К3, на 

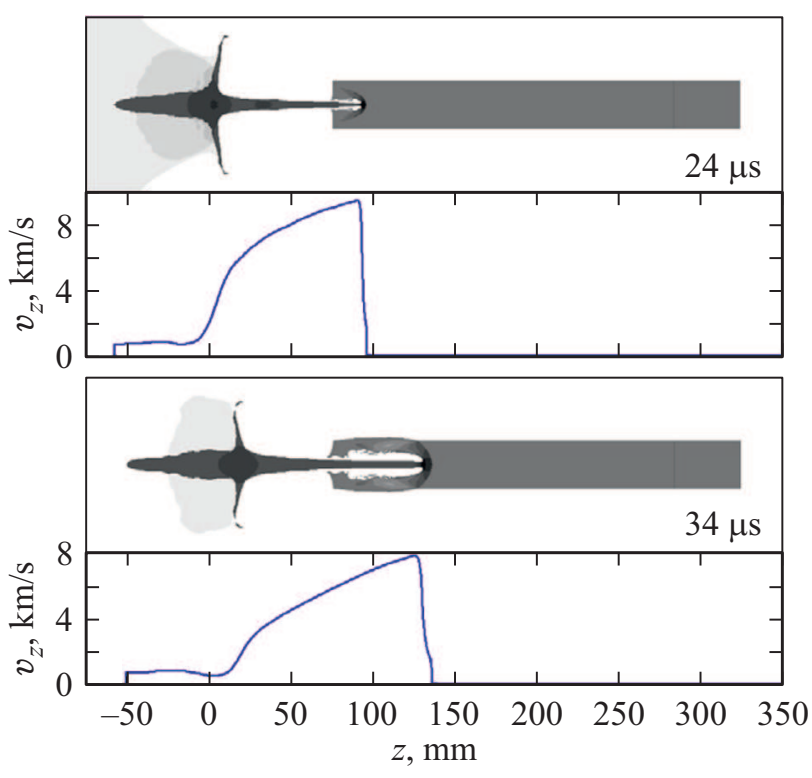

Рис. 7. Пробитие КС свинцового стержня-отсекателя длиной $2.5 d_{0}$ (время отсчитывается от момента инициирования КЗ).

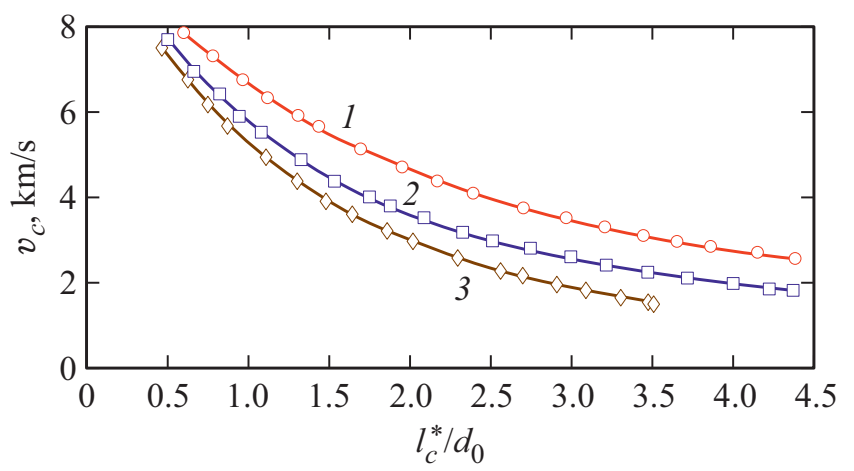

Рис. 8. Зависимость скорости лидирующего элемента сохраняющейся хвостовой части КС от пути, пройденного КС в стержнях-отсекателях длиной $4.5 d_{0}$ из разных материалов: 1 сталь; 2 - свинец; 3 - тяжелый сплав ВНЖ-90.

котором происходит разрыв на отдельные безградиентные элементы хвостовых участков КС со скоростями в диапазоне $2-3 \mathrm{~km} / \mathrm{s}$, в подавляющем большинстве случаев превышает $5 d_{0}$. Отсюда следует, что использование стержней-отсекателей из свинца или сплава ВНЖ-90 позволяет провести эксперименты по сравнительному анализу пробивного действия хвостовых участков КС в неразорванном и разорванном состояниях, о которых говорилось выше.

Следует отметить, что дистанция „отсечки“ хвостовой части КС с использованием стержней-отсекателей, по всей видимости, может быть несколько сокращена, если
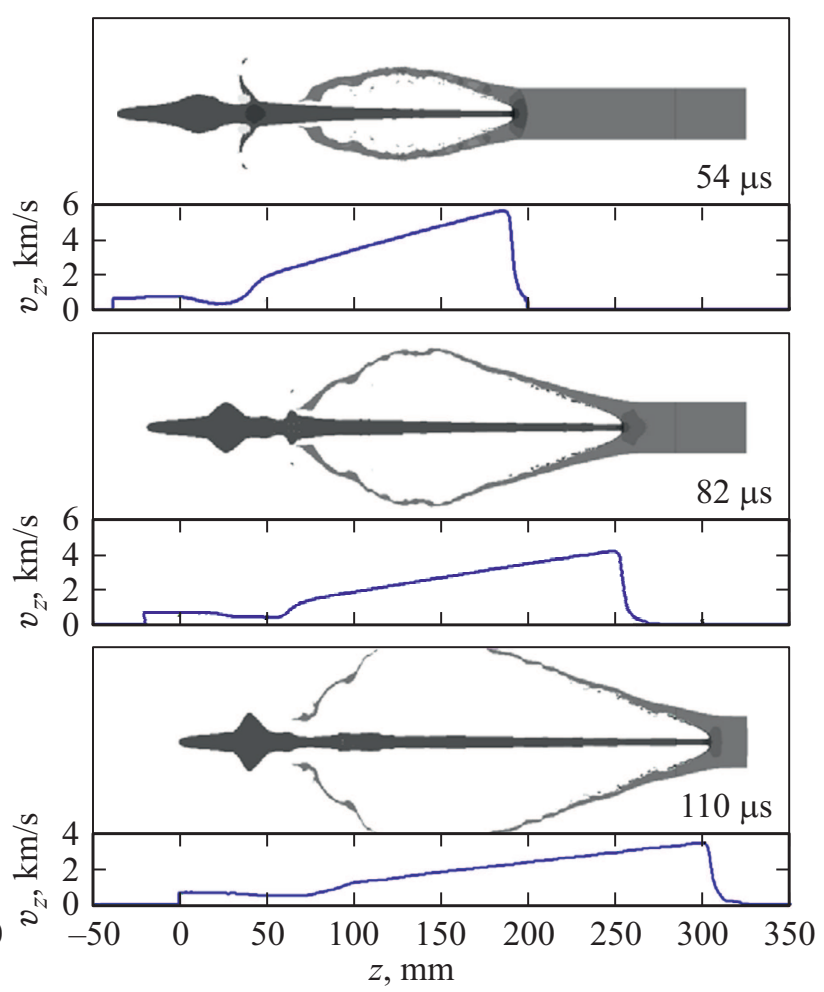

стержень приблизить к КЗ до расстояния примерно $h_{c}=0.5 d_{0}$ (рис. 5). При этом стержень лучше брать в форме усеченного конуса с уменьшенным диаметром основания, обращенного к КЗ. Это должно увеличить скорость радиального разлета материала лицевой части стержня во избежание его возможного столкновения с еще не схлопнувшимся основанием кумулятивной облицовки. Как видно из рис. 6 и 7, материал ближнего к КЗ основания стержня разлетается медленнее всего (однако нужно учитывать, что расчеты были проведены без учета в модели поведения материала стержня возможности его разрушения, которое в реальной ситуации с неизбежностью будет происходить).

Ясно, что окончательные выводы по возможности использования стержней-отсекателей для экспериментального исследования пробивного действия хвостовых участков КС, а также по выбору их параметров для сохранения „хвоста“ струи нужной длины могут быть сделаны только по результатам соответствующих экспериментов. Однако не видится каких-либо серьезных причин для существенного расхождения этих результатов с результатами проведенного численного моделирования.

\section{Заключение}

Предложенный способ „отсечки“ более скоростной части КС с использованием стержней-отсекателей позволяет провести детальное экспериментальное исследование пробивной способности хвостовых участков КС с 
определением критической скорости струи и динамической твердости материала преграды в условиях проникания КС. Результаты этого исследования представляют интерес как в фундаментальном, так и в прикладном отношении. Достоверная информация по значениям динамической твердости преград из различных материалов в условиях проникания КС необходима для расчетного прогнозирования пробивного действия КЗ. Основное прикладное предназначение предложенных экспериментальных исследований - проверить возможность предсказываемого расчетами существенного увеличения пробивного действия КЗ за счет повышения точности их изготовления и обусловленного этим снижения эффективной скорости КС. Принципиальная возможность регулируемой „отсечки“ стержнями-отсекателями более скоростной части КС, предшествующей хвостовой, подтверждена результатами численного моделирования.

\section{Благодарности}

Автор выражает большую благодарность О.В. Свирскому за полезное обсуждение рассмотренных в работе вопросов.

\section{Конфликт интересов}

Автор заявляет, что у него нет конфликта интересов.

\section{Список литературы}

[1] М.А. Лаврентьев. Успехи математических наук, 12 (4), 41 (1957).

[2] Физика взрыва, под ред. Л.П. Орленко. (Физматлит, М., 2004), т. 2.

[3] W.P. Walters, J.A. Zukas. Fundamentals of Shaped Charges (Wiley, NY., 1989)

[4] P.C. Chou, W.J. Flis. Propellants, Explosives, Pyrotechnics, 11 (4), 99 (1986).

[5] H. Shekhar. Central Europ. J. Energetic Mater., 9(2), 155 (2012).

[6] W.P. Walters, R.L. Summers. Propellants, Explosives, Pyrotechnics, 18 (5), 241 (1993).

[7] E. Hennequin. Propellants, Explosives, Pyrotechnics, 21 (4), 181 (1996).

[8] J. Petit. J. Appl. Phys., 98 (12), 123521 (2005).

[9] O. Ayisit. Intern. J. Impact Eng., 35 (12), 1399 (2008).

[10] E. Hirsch. Propellants, Explosives, Pyrotechnics, 6 (4), 104 (1981).

[11] О.В. Свирский, М.А. Власова, Т.А. Торопова, А.И. Нечаев, В.А. Крутяков. Труды Межсунар. конф. „V Харитоновские тематические научные чтения" (ВНИИЭФ, Саров, 2003), с. 473.

[12] R. Cornish, J.T. Mills, J.P. Curtis, D. Finch. Intern. J. Impact Eng., 26 (1-10), 105 (2001).

[13] Q.-Q. Xiao, Z.-X. Huang, X.-D. Zu, X. Jia. Propellants, Explosives, Pyrotechnics, 41 (1), 76 (2016).

[14] M. Moyses. Proc. of the 17th Intern. Symp. on Ballislics (Midrand, South Africa, 1998), vol. 2, p. 413.

[15] M. Held. Propellants, Explosives, Pyrotechnics, 13 (4), 113 (1988).
[16] D. Boeka, S. Hancock, N. Ouye. Proc. of the 19th Intern. Symp. on Ballislics (Interlaken, Switzerland, 2001), vol. 3, p. 1471.

[17] S.L. Hancock. Intern. J. Impact Eng., 26 (1-10), 289 (2001).

[18] О.В. Свирский, М.А. Власова. Физика горения и взрыва, 55 (6), 115 (2019). [O.V. Svirsky, M.A. Vlasova. Combustion, Explosion, and Shock Waves, 55 (6), 739 (2019).]

[19] Частные вопросы конечной баллистики, под ред. В.А. Григоряна (Изд-во МГТУ им. Н.Э. Баумана, М., 2006)

[20] Л.П. Орленко. Поведение материалов при интенсивных динамических нагрузках (Машиностроение, М., 1964)

[21] Методы исследования свойств материалов при интенсивных динамических нагрузках, под ред. М.В. Жерноклетова (ФГУП „РФЯЦ-ВНИИЭФ“, Саров, 2005)

[22] P.C. Chou, M. Grudza, Y.F. Liu, Z. Ritman. Proc. of the 13th Intern. Symp. on Ballislics (Stockholm, Sweden, 1992), vol. 2, p. WM/489.

[23] P.Y. Chantaret. Proc. of the 17th Intern. Symp. on Ballislics (Midrand, South Africa, 1998), vol. 2, p. 373.

[24] O.V. Svirsky, M.A. Vlasova, M.I. Korotkov, V.A. Krutyakov, T.A. Toropova. Intern. J. Impact Eng., 29 (1-10), 683 (2003).

[25] M. Mayseless, E. Hirsch, A. Lindenfeld, Y. Me-Bar. Proc. of the 17th Intern. Symp. on Ballislics (Midrand, South Africa, 1998), vol. 2, p. 187.

[26] С.В. Федоров, В.А. Велданов. ЖТФ, 76 (7), 134 (2006). [S.V. Fedorov, V.A. Veldanov. Tech. Phys., 51 (7), 952 (2006).]

[27] С.В. Федоров. ЖТФ, 77 (10), 131 (2007). [S.V. Fedorov. Tech. Phys., 52 (10), 1379 (2007).]

[28] С.В. Федоров, В.А. Велданов. ЖТФ, 83 (2), 15 (2013). [S.V. Fedorov, V.A. Veldanov. Tech. Phys., 58 (2), 165 (2013).]

[29] С.В. Федоров. Вестник МГТУ им. Н.Э. Баумана. Сер. Естественные науки, (3), 71 (2017). DOI: 10.18698/18123368-2017-3-71-92 [S.V. Fedorov. Herald of the Bauman Moscow State Tech. Univ., Nat. Sci., (3), 71 (2017). DOI: 10.18698/1812-3368-2017-3-71-92]

[30] А.В. Бабкин, В.И. Колпаков, В.Н. Охитин, В.В. Селиванов. Прикладная механика сплошных сред. Т. 3. Численные методы в задачах физики быстропротекающих процессов (Изд-во МГТУ им. Н.Э. Баумана, М., 2006)

[31] С.В. Федоров. Физика горения и взрыва, 52 (5), 116 (2016). [S.V. Fedorov. Combustion, Explosion, and Shock Waves, $52(5), 600$ (2016).]

[32] Г.Б. Черняк, К.Б. Поварова. Вольфрам в боеприпасах (ЦНИИХМ, М., 2014)

[33] S.V. Fedorov, Ya.M. Bayanova. Proc. of the 25th Intern. Symp. on Ballislics (Beijing, China, 2010), p. 1032.

[34] С.В. Федоров, А.В. Бабкин, В.А. Велданов, Н.А. Гладков, С.В. Ладов. Вестник МГТУ им. Н.Э. Баумана. Сер. Естественные науки, (5), 18 (2016). DOI: 10.18698/1812-33682016-5-18-32 [S.V. Fedorov, A.V. Babkin, V.A. Veldanov, N.A. Gladkov, S.V. Ladov. Herald of the Bauman Moscow State Tech. Univ., Nat. Sci., (5), 18 (2016). DOI: 10.18698/1812-3368-2016-5-18-32]

[35] J.P. Curtis, M. Moyses, A.J. Arlow, K.G. Kowan. Proc. of the 16th Intern. Symp. on Ballislics (San Francisco, USA, 1996), vol. 2, p. WM/369. 